\title{
Stationary and oscillatory patterns in a coupled Brusselator model
}

\author{
Roumen Anguelov, Stephanus Marnus Stoltz \\ Department of Mathematics and Applied Mathematics, University of Pretoria \\ Pretoria 0002, South Africa
}

\begin{abstract}
This paper presents a numerical investigation into the pattern formation mechanism in the Brusselator model focusing on the interplay between the Hopf and Turing bifurcations. The dynamics of a coupled Brusselator model is studied in terms of wavelength and diffusion, thus providing insight into the generation of stationary and oscillatory patterns. The expected asymptotic behaviour is confirmed by numerical simulations. The observed patterns include inverse labyrinth oscillations, inverse hexagonal oscillations, dot hexagons and parallel lines. Keywords: Nonlinear reaction rate, Brusselator model, coupled system, Turing patterns, Hopf bifurcation

2010 MSC: 35B36, 35K57, 80A30, 92C45, 92E20
\end{abstract}

\section{Introduction}

Models involving termolecular reaction steps exhibit interesting properties and pose challenging mathematical problems regarding the asymptotic behaviour of the solutions. It is well-known that models of reaction sequences with two intermediates and only uni- and bimolecular steps do not admit limit cycles [3, Section 7.1]. Therefore, for instability to occur in the thermodynamic branch (the solution in equilibrium) one needs to use cubic reaction rates [1], [8], [3].

The following reaction sequence was studied by Prigogine and Lefever in 1968 [5]:

Email addresses: roumen.anguelov@up.ac.za (Roumen Anguelov), stoltzstep@gmail.com (Stephanus Marnus Stoltz) 


$$
\begin{gathered}
\mathrm{A} \underset{k_{-1}}{\stackrel{k_{1}}{\rightleftharpoons}} \mathrm{U} \\
\mathrm{B}+\mathrm{U} \underset{k_{-2}}{\stackrel{k_{2}}{\rightleftharpoons}} \mathrm{V}+\mathrm{D} \\
2 \mathrm{U}+\mathrm{V} \underset{k_{-3}}{\stackrel{k_{3}}{\rightleftharpoons}} 3 \mathrm{U} \\
\mathrm{U} \underset{k_{-4}}{\stackrel{k_{4}}{\rightleftharpoons}} \mathrm{E}
\end{gathered}
$$

We note that the third step in the sequence involves a cubic nonlinear reaction term. Under the assumptions that

(i) D and E are removed from the reaction domain the instant they are produced (or equivalently, $\left.k_{-2}=k_{-4}=0\right)$,

(ii) the nonlinear reaction is irreversible $\left(k_{-3}=0\right)$,

(iii) $\mathrm{A}$ is in sufficient abundance,

the dynamics of the reaction sequence is represented in [5] by two rate equations:

$$
\begin{aligned}
& \frac{\partial U}{\partial \hat{t}}=k_{1} A-\left(k_{2} B+k_{4}\right) U+k_{3} U^{2} V+\hat{D}_{u} \nabla^{2} U \\
& \frac{\partial V}{\partial \hat{t}}=k_{2} B U-k_{3} U^{2} V+\hat{D}_{v} \nabla^{2} V
\end{aligned}
$$

By scaling of the variables,

$$
\begin{array}{ccc}
t=k_{4} \hat{t} & u=\left(\frac{k_{3}}{k_{4}}\right)^{\frac{1}{2}} U & v=\left(\frac{k_{3}}{k_{4}}\right)^{\frac{1}{2}} V \\
a=\left(\frac{k_{1}^{2} k_{3}}{k_{4}^{3}}\right)^{\frac{1}{2}} A & b=\frac{k_{2}}{k_{4}} B & D_{i}=\frac{\hat{D}_{i}}{k_{4}}
\end{array}
$$

the model (1) is simplified to the following model involving only two parameters.

$$
\begin{aligned}
& \frac{\partial u}{\partial t}=a-(b+1) u+u^{2} v+D_{u} \nabla^{2} u \\
& \frac{\partial v}{\partial t}=b u-u^{2} v+D_{v} \nabla^{2} v
\end{aligned}
$$


The system (3) of reaction diffusion partial differential equations is known as the trimolecular model or the Brusselator model, the latter term coined by Tyson in 1973 [7]. This model has been widely used to illustrate and study basic features of chemical reaction models involving trimolecular steps. In some sense it plays in the settings of these models a pivotal role similar to the role the harmonic oscillator and the Heisenberg model play in ferromagnetism [3].

This paper presents a numerical investigation into the pattern formation mechanism in the Brusselator model. The next section (Section 2) is devoted to studying the interplay between the two bifurcations in the model, namely the Hopf bifurcation and the Turing bifurcation. This investigation is largely motivated by the observations in [1] that Turing patterns eventually (for sufficiently small ratio of the diffusion coefficients) dominate the Hopf bifurcation induced oscillations. The numerical simulations yield a hyperbola-like shaped boundary between the two regions. Oscillatory patterns are observed only in a small area near the horizontal part of curve. Based on these results, Section 3 deals with pattern formation in a coupled Brusselator model, that is, two systems of the form (3) linked via linear interaction terms. The study of the dynamics of this model in terms of wavelength and diffusion provides insight into generation of stationary and oscillatory patterns. The expected asymptotic behaviour is confirmed by numerical simulations. The observed patterns include inverse labyrinth oscillations, inverse hexagonal oscillations, dot hexagons and parallel lines. In Section 4 we provide some concluding remarks and directions for future work. For completeness of the exposition, details on the numerical method used for the simulations are presented in the Appendix.

\section{Turing and Hopf bifurcations in the Brusselator model}

The system (3) has one spatially homogeneous steady state, $u^{*}=a, v^{*}=\frac{b}{a}$. Its stability is influenced by two factors: the appearance of spatially homogeneous limit cycle (Hopf bifurcation) and the ratio of the diffusion coefficients (Turing instability). We recall them briefly. 
The spatially homogeneous solutions of (3) satisfy the system of ODEs

$$
\begin{aligned}
& \frac{d u}{d t}=a-(b+1) u+u^{2} v \\
& \frac{d v}{d t}=b u-u^{2} v .
\end{aligned}
$$

Linear stability analysis yields that $\left(u^{*}, v^{*}\right)$ is an asymptotically stable equilibrium of (4) if $b<a^{2}+1$ with Hopf bifurcation at $b=a^{2}+1$. This means that for $b>a^{2}+1$ the equilibrium is unstable and all nonequilibrium solutions approach a stable limit cycle.

Turing instability refers to the fact that the steady state $\left(u^{*}, v^{*}\right)$ is unstable when the ratio of the diffusion coefficients is sufficiently small/large. Following the standard approach, e.g. as presented in [2], we obtain the Turing instability conditions in the form,

$$
\begin{aligned}
& b<b_{H}:=a^{2}+1 \\
& b>b_{T}:=\left(1+a \sqrt{\frac{D_{u}}{D_{v}}}\right)^{2}
\end{aligned}
$$

We note that (5) and (6) hold simultaneously only if $\frac{D_{u}}{D_{v}}<1$, which implies that the activator, $u$, diffuses slower than the inhibitor, $v$. Further, for any fixed values of $a$ and $b>1$, stable patterns are formed when $\frac{D_{u}}{D_{v}}$ is sufficiently small so that $b>b_{T}$. Therefore, in the subcritical Hopf parameter domain given by (5) we have two qualitatively different options for the dynamics of the model (3): (i) stable spatially homogenous steady state $\left(u^{*}, v^{*}\right)$ and (ii) formation of stable spatial patterns, with the respective parameter subdomains separated by the bifurcation line $b=b_{T}$.

Our main interest is in the asymptotic properties of the model (3) in the supercritical Hopf domain $b>b_{H}$. In this parameter domain the model (3) has a spatially homogeneous limit cycle, so called bulk oscillations, corresponding to the limit cycle of (4). We investigate numerically the asymptotic behavior of the solutions of $(3)$ with a focus on the disappearing of oscillations and formation of stable patterns when $\frac{D_{u}}{D_{v}}$ is sufficiently small. The numerical method used for the simulations is presented in the Appendix. The system (3) is considered 
for $x \in[0,200] \times[0,200]$ with periodic boundary conditions

$$
\begin{gathered}
\left.u\right|_{x_{1}=0}=\left.u\right|_{x_{1}=200},\left.\quad u\right|_{x_{2}=0}=\left.u\right|_{x_{2}=200}, \\
\left.v\right|_{x_{1}=0}=\left.u\right|_{x_{1}=200},\left.\quad v\right|_{x_{2}=0}=\left.u\right|_{x_{2}=200} .
\end{gathered}
$$

Following the approach in [11, we fix the values of $D_{v}$ and $a$ and vary $b$ and $D_{u}$. More precisely, we consider $b \in[7.2,13.2], D_{u} \in[3,10]$ with independent increments of 0.25 each, while keeping $D_{v}=10$ and $a=3$. The system (3) is solved with initial conditions which are random perturbations of the spatially homogeneous steady state. The points of the parameter grid are given in Figure 1. At any parameter point, irrespective of the initial condition, the computed solution eventually settles in one of the following stable states: spatially homogeneous steady state, bulk oscillations, oscillating patterns or stationary Turing patterns. Different markers in the Figure 1 indicate which one of these asymptotic behaviors is observed (see Legend). Interactive form of this figure is available on [6]. Clicking a marker on the diagram plays a video of a typical evolution of the solution for the respective values of the parameters $b$ and $D_{u}$.

Below the Hopf bifurcation line $b=b_{H}$, and as expected, the line $b=b_{T}$ separates a stable steady state region and a Turing patterns region. Interestingly, above the line $b=b_{H}$, there is a very well pronounced separation line $b=b_{T^{*}}$ between the bulk oscillations region and the pattern formation region. Mostly, the patterns are stationary. The exception is a narrow area between the lines $b=b_{H}$ and $b=b_{T^{*}}$, where oscillating patterns occur. Schematically the different regions and dividing lines are presented in Figure 2. The line $b=b_{T^{*}}$ which separates the bulk oscillations region and the pattern formation region has a hyperbola-like shape. This implies that for any fixed $b>b_{H}$, a stable stationary pattern is formed provided the fraction $\frac{D_{u}}{D_{v}}$ is sufficiently small. Further, the simulations indicate a possible vertical asymptote at a value of, $D_{u}$ in $(3.4,3.6)$, that is for $D_{u}$ smaller than this value a stable pattern is formed irrespective of the value of $b$. 


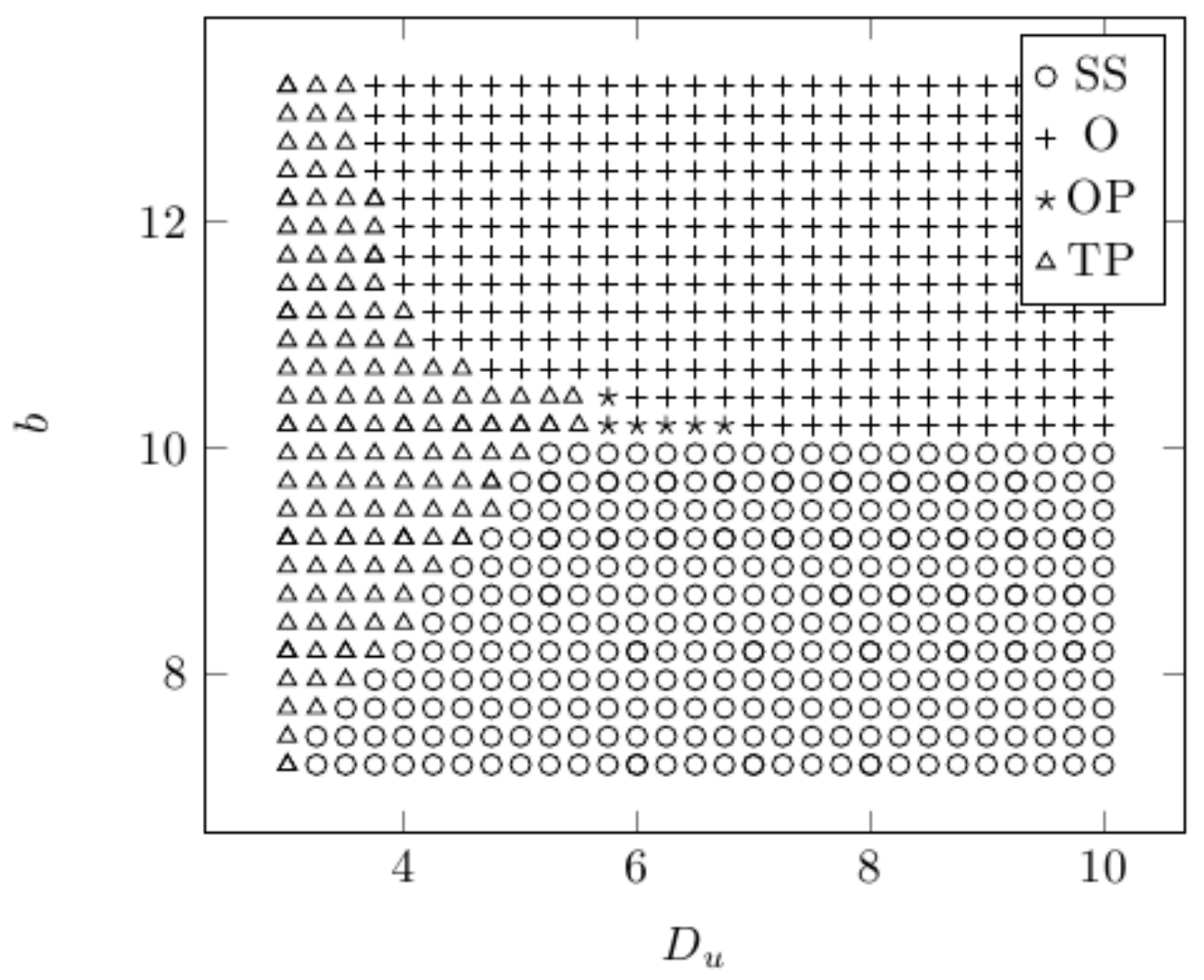

Figure 1: Classification of the numerical simulations of the Brusselator model into the following classes: SS - Stable stationary state, O - Bulk Oscillations, OP - Oscillating patterns, TP - Turing patterns. Interactive form of the figure is available in [6]

\section{The coupled Brusselator model}

The coupled Brusselator model comprises two systems of the form (3) coupled via linear reaction terms. Its general form is

$$
\begin{aligned}
& \frac{\partial u_{i}}{\partial t}=D_{u_{i}} \nabla^{2} u_{i}+\alpha\left(u_{j}-u_{i}\right)+f\left(u_{i}, v_{i}\right) \\
& \frac{\partial v_{i}}{\partial t}=D_{v_{i}} \nabla^{2} v_{i}+\beta\left(v_{j}-v_{i}\right)+g\left(u_{i}, v_{i}\right)
\end{aligned}
$$

with $i, j=1,2, i \neq j$. The reactions $f$ and $g$ are as in (3), namely $f(u, v)=a-(1+b) u+u^{2} v$ and $g(u, v)=b u-u^{2} v$. The system (9) can be considered as a model of the reaction sequences in two thin layers of gel that meet at an interface. Each layer contains the same set of 


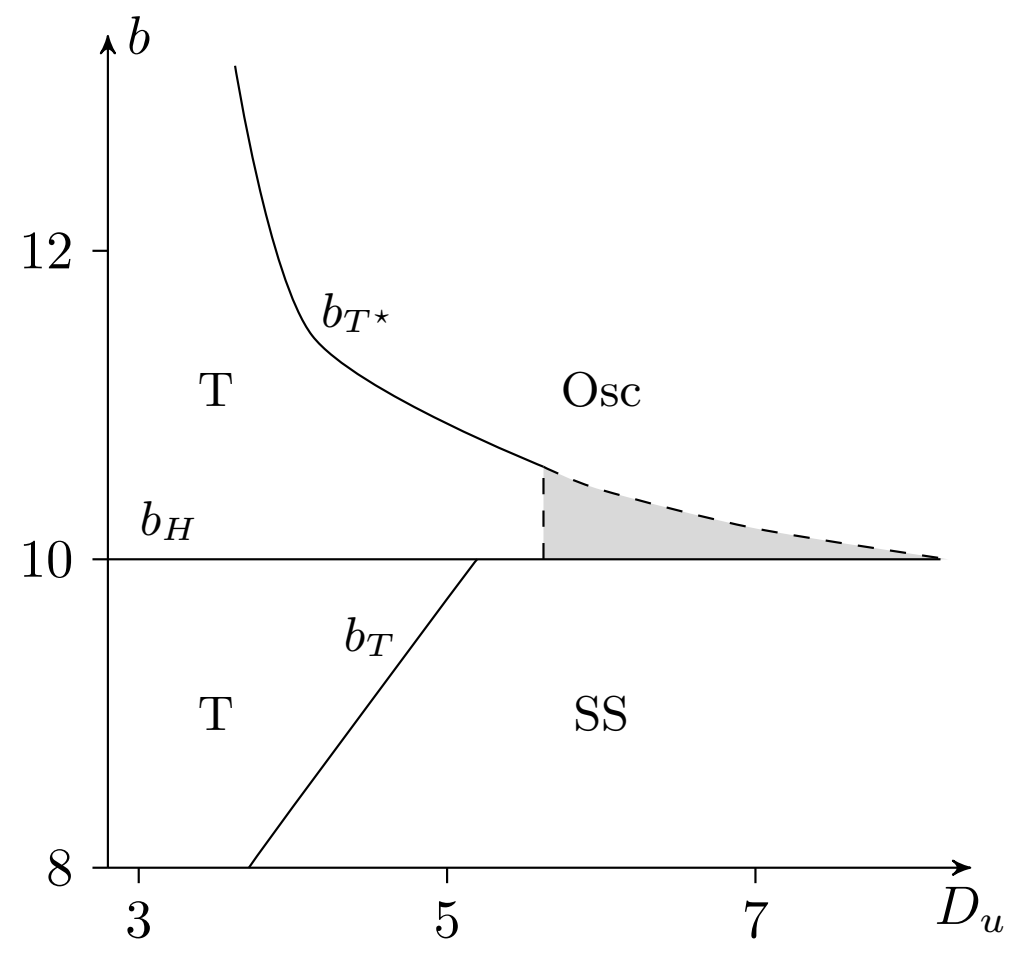

Figure 2: Schematic representation of the regions in Figure 1. T - stable Turing patterns, Osc bulk oscillations, SS: stable steady state

reactants with the same kinetics but with different diffusion parameters. The difference in diffusion can be owed to either physical (viscosity or density of the gel) or chemical (complex formation) factors. We note that the model represents two diffusion processes, referred to as horizontal and vertical diffusion. Horizontal diffusion is the diffusion in the two dimensional spatial domain of one layer and is described by the Laplacian term in each equation of (9). The vertical diffusion is the interaction between the two layers represented by the linear terms $\alpha\left(u_{j}-u_{i}\right)$ and $\beta\left(v_{j}-v_{i}\right), i, j=1,2, i \neq j$. Yang et al. [10] studied the stable spatial resonance and superposition patterns in this coupled system. The spatial resonance of two wavelengths in the interacting layers produce stationary patterns, known as "black/white-eyes". Whereas the superposition patterns combines stripes and/or spots of varying size layered on top of each other. They also report a three-phase oscillatory 


\begin{tabular}{cccccccccc}
\hline \hline Figure & $a$ & $b$ & $\alpha$ & $D_{u 1}$ & $D_{v 1}$ & $D_{u 2}$ & $D_{v 2}$ & $k_{1}$ & $k_{2}$ \\
\hline $3 \mathrm{a}$ & 3 & 10.2 & 0.1 & 4 & 10 & 10 & 10 & 0.83 & 0.1 \\
3b & 3 & 10.2 & 0.1 & 5 & 10 & 10 & 10 & 0.68 & 0.1 \\
$3 \mathrm{c}$ & 3 & 13.2 & 0.1 & 3 & 10 & 10 & 10 & 1.25 & 0.4 \\
\hline 4bI & 3 & 10.2 & 1 & 6 & 10 & 30 & 100 & 0.56 & 0.33 \\
$4 \mathrm{bII}$ & 3 & 10.2 & 1 & 6 & 10 & 37 & 100 & 0.56 & 0.28 \\
$4 \mathrm{bIII}$ & 3 & 10.2 & 1 & 6 & 10 & 41 & 100 & 0.56 & 0.26 \\
$4 \mathrm{bIV}$ & 3 & 10.2 & 1 & 6 & 10 & 44 & 100 & 0.56 & 0.24 \\
\hline \hline
\end{tabular}

Table 1: Parameters used in simulations of coupled Brusselator model $(\alpha=\beta)$

interlacing hexagonal lattice pattern,known as "twinkling-eye" pattern, which occurs due to resonance between a Turing mode and its subharmonic.

Here we show a mechanism of generating oscillating patterns in the coupled model which is based on the asymptotic properties of a single layer model as presented in Figure 2. More precisely, oscillating patterns are obtained by coupling a layer with bulk oscillations and a layer with a Turing pattern. Roughly speaking, the one layer provides patterns while the other one drives the oscillations. It is important to observe that due to the shape of the curve $b=b_{T^{*}}$ in Figure 2 one can indeed obtain two layers of such different properties by just varying $D_{u}$. This property is essential since the two coupled layers in (9) differ only in their diffusion coefficients.

The parameters used in our simulations are given in Table 1. In the first set of simulations we varied the diffusion $D_{u_{1}}$ in the first layer while keeping the rest of the parameters fixed. We observe an oscillating pattern when we couple a strong bulk oscillating layer with a Turing pattern layer, see Figure $3 \mathrm{a}$ and Figure $3 \mathrm{~b}$. Typical Turing patterns observed in a single layer Brusselator model (Figure 4a) oscillate through inverting the concentration of each previous time step. Increasing $b$ leads to a temporal separation of the Turing patterns and the bulk oscillations with the system spending longer periods in a set pattern. 
I: $\mathbf{T}$

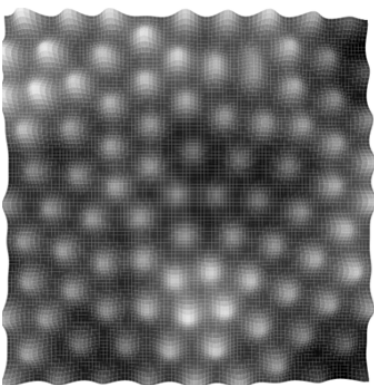

I: $\mathbf{T}$

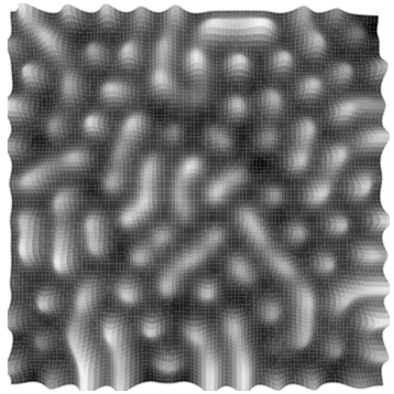

II: $\mathbf{T}+\mathbf{1}$

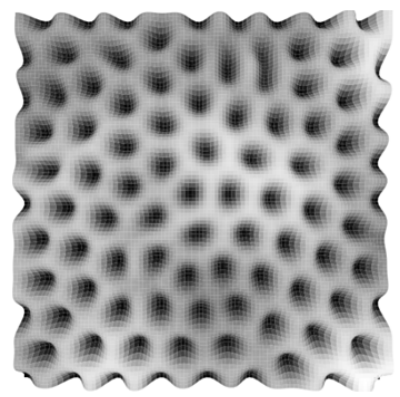

III: $\mathbf{T}+2$

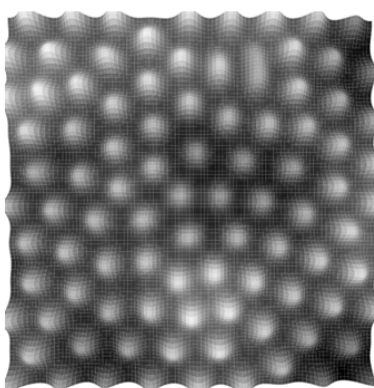

(a)
III: $\mathbf{T}+2$

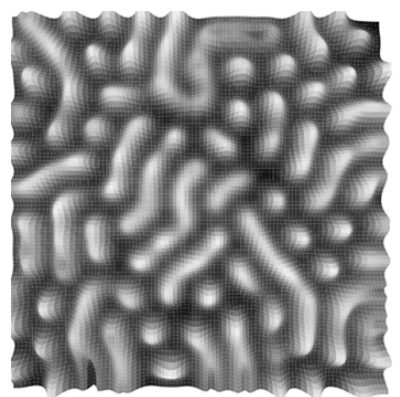

IV: $\mathbf{T}+\mathbf{3}$

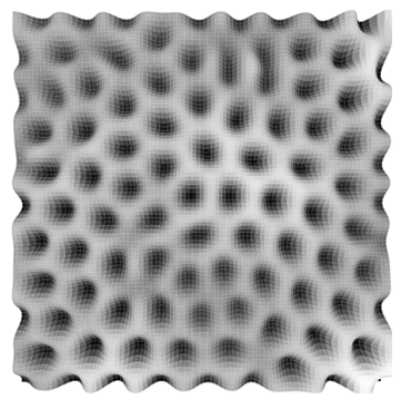

IV: $\mathbf{T}+3$

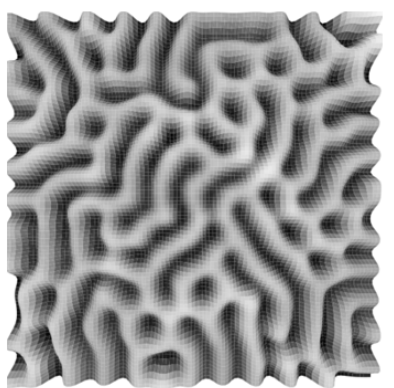

(b)

Figure 3: Oscillating pattern in a coupled Brusselator model (a) Honeycomb-inverse pattern oscillations (b) Labrynth-inverse pattern oscillations

The second set of simulations shows deviation from the typical Turing patterns of a single layer, Figure $4 \mathrm{~b}$, due to the interacting Turing modes on different scales. The wavenumber was derived from the linearized system in the form

$$
k_{i}=\sqrt{\frac{1}{2}\left(\frac{f_{u}}{D_{u}}+\frac{g_{v}}{D_{v}}\right)}=\sqrt{\frac{1}{2}\left(\frac{b-1}{D_{u}}-\frac{a^{2}}{D_{v}}\right)} .
$$

We kept the short wavelengths fixed (wavenumber $k_{1}=0.56$ ) while varying the long wavelengths. The long wavelength was achieved by increasing both diffusion coefficients $D_{u_{2}}$ and $\left.D_{v_{2}}\right)$, thus providing for the layer to remain in the Turing domain. The stationary patterns progress from Labyrinth patterns through to Dot Hexagonal patterns with Parallel lines occurring when the wavelength ratio $\frac{k_{1}}{k_{2}}=2$. Hexagons first appear when the wavelength ratio $\frac{k_{1}}{k_{2}}>2$. When $\frac{k_{1}}{k_{2}}>2.3$, we observe a clear dot hexagonal pattern, as seen in Figure 
I: Hexagonal

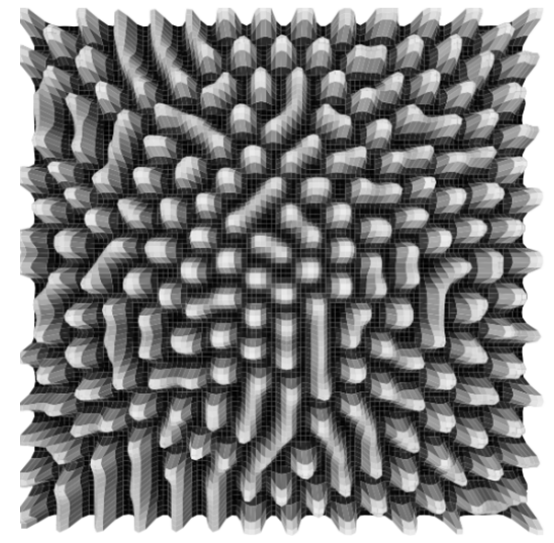

II: Labyrinth

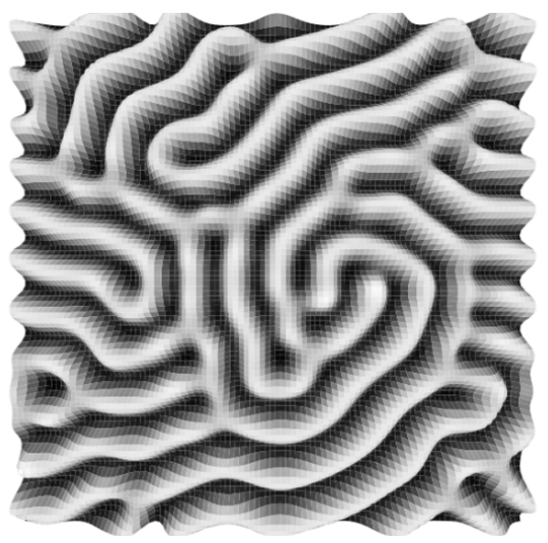

III: Honeycomb

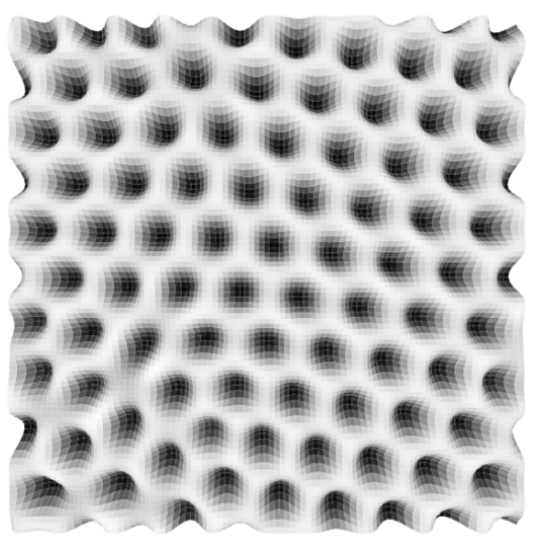

(a)

\section{I: Labyrinth}

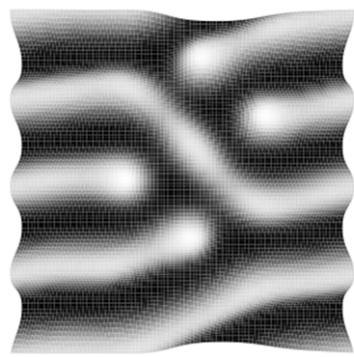

\section{II: Parallel}

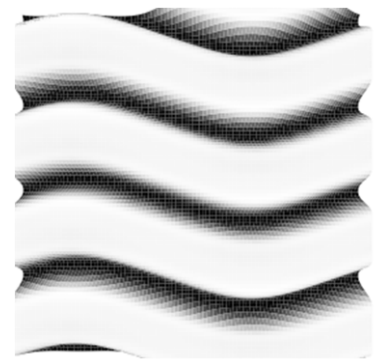

III: Par. Hexagons

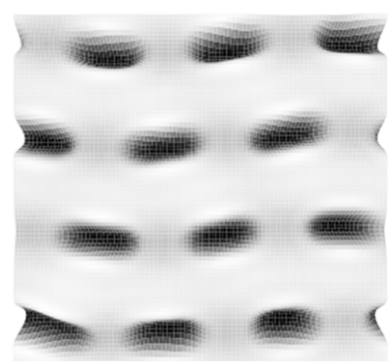

(b)

\section{IV: Dot Hexagons}

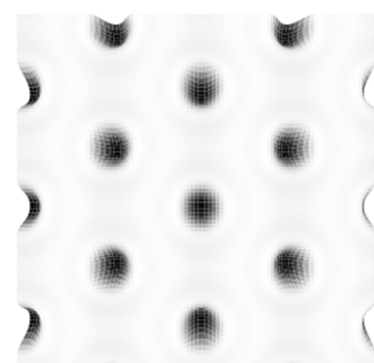

Figure 4: Stationary patterns in the coupled Brusselator model: (a) typical patterns observed in a single layer Brusselator model; (b) patterns that occur due to interaction of two Turing modes with various wavelengths

4biII.

\section{Conclusion}

The numerical investigation in this paper provides insight into the asymptotic behavior of the solutions of a single layer Brusselator model, characterizing the parameter region for each of the three qualitatively different cases. Particular attention is given to the supercritical Hopf bifurcation parameter domain where no substantial theory is available. The obtained results are used further in revealing an essential mechanism generating oscillating patterns 
in the coupled Brusselator model. Future work is envisaged on the analysis of the single and coupled model using a theoretical approach similiar to Rashkov's [12], with the aim of producing a more complete classification of the stationary and oscillating patterns as well as their generating mechanisms.

\section{Appendix}

The Alternating-Direction Implicit method (ADI) uses the concept of operator splitting or time splitting. The idea is to divide each timestep into two steps of size $\Delta t / 2$, where in each substep, a different dimension is treated implicitly. This leads to a set of equations for each substep similiar to the set of equations for the implicit one dimensional case. The Peaceman-Rachford variant [4] of the ADI method consists of solving (9), with the prediction substep $(\Delta t / 2)$ using the backward Euler method for the $x$ derivative terms and the forward Euler method for the $y$ derivative terms. The correction substep $(\Delta t)$ then proceeds to swap the Euler methods around using the forward Euler method for $x$ derivative terms and the backward Euler method for the $y$ derivative terms. We illustrate the substeps below,

$$
\begin{aligned}
& \frac{u_{l, m}^{n+\frac{1}{2}}-u_{l, m}^{n}}{\Delta t / 2}=D_{u}\left(\frac{u_{l+1, m}^{n+\frac{1}{2}}-2 u_{l, m}^{n+\frac{1}{2}}+u_{l-1, m}^{n+\frac{1}{2}}}{\Delta x^{2}}+\frac{u_{l, m+1}^{n}-2 u_{l, m}^{n}+u_{l, m+1}^{n}}{\Delta y^{2}}\right)+F\left(u_{l, m}^{n}, v_{l, m}^{n}\right) \\
& \frac{u_{l, m}^{n+1}-u_{l, m}^{n+\frac{1}{2}}}{\Delta t / 2}=D_{u}\left(\frac{u_{l+1, m}^{n+\frac{1}{2}}-2 u_{l, m}^{t+\frac{1}{2}}+u_{l-1, m}^{n+\frac{1}{2}}}{\Delta x^{2}}+\frac{u_{l, m}^{n+1}-2 u_{l, m}^{n+1}+u_{l, m+1}^{n+1}}{\Delta y^{2}}\right)+F\left(u_{l, m}^{n+\frac{1}{2}}, v_{l, m}^{n+\frac{1}{2}}\right)
\end{aligned}
$$

where $F(u, v)=\alpha\left(u_{j}-u_{i}\right)+f\left(u_{i}, v_{i}\right)$, we note that the same holds for $v$.

Using periodic boundary conditions for the system (9) the first step in (10) is reduced 
to solving for $m=1,2, \ldots, M$, a linear tridiagonal cyclic system of the form,

$$
\left(\begin{array}{ccccccc}
1+2 r & -r & 0 & \ldots & & & -r \\
-r & 1+2 r & -r & \ldots & & & \\
& & & \ldots & & & \\
& & & \ldots & -r & 1+2 r & -r \\
-r & & & \ldots & 0 & -r & 1+2 r
\end{array}\right) \cdot\left(\begin{array}{c}
u_{1, m}^{n+\frac{1}{2}} \\
u_{2, m}^{n+\frac{1}{2}} \\
\ldots \\
u_{L-1, m}^{n+\frac{1}{2}} \\
u_{L, m}^{n+\frac{1}{2}}
\end{array}\right)=\left(\begin{array}{c}
f_{1} \\
f_{2} \\
\ldots \\
f_{L-1} \\
f_{L}
\end{array}\right)
$$

where $r=\frac{D_{u} \Delta t}{2 \Delta x^{2}}, s=\frac{D_{u} \Delta t}{2 \Delta y^{2}}, f_{i}=-s u_{i-1, m}+(1+2 s) u_{i, m}-s u_{i+1, m}+\frac{\Delta t}{2} F\left(u_{i, m}^{n}, v_{i, m}^{n}\right), i=$ $1,2, \ldots, L$. The second step in 10 is implemented similarly by interchanging $x$ and $y$. In order to solve the systems efficiently we use the Sherman-Morrison formula [9, Section 2.7.1], handling the coefficient matrix as a tridiagonal matrix plus a correction. This requires us to define the following two vectors. Let $P$ be the tridiagonal matrix in (11),

$$
\varphi_{1}=\left(\begin{array}{c}
\gamma \\
0 \\
\vdots \\
0 \\
p_{L, M}
\end{array}\right) \quad \varphi_{2}=\left(\begin{array}{c}
1 \\
0 \\
\vdots \\
0 \\
p_{1,1} / \gamma
\end{array}\right)
$$

where $\gamma=-p_{1,2}$. We can now solve the tridiagonal matrix with the following steps:

(i) Solve $P^{\prime} \cdot u=f$

(ii) Set up the vector $\varphi_{1}$.

(iii) Solve $P^{\prime} \cdot z=\varphi_{1}$.

(iv) Obtain the form, $\Psi=\varphi_{2} \cdot u_{m} /\left(1+\varphi_{2} \cdot z\right)$

(v) Use the form $\Psi$ to obtain the new values of $u_{m}$, i.e $u_{m}=u_{m}-\Psi \cdot z$ where $P^{\prime}=P$ but with the following two terms modified.

$$
p_{1,2}^{\prime}=p_{1,2}-\gamma \quad p_{L, M}^{\prime}=p_{L, M}-p_{L, M} \frac{p_{1,1}}{\gamma}
$$




\section{Acknowledgement}

This work has been supported by the SARChI Chair in Mathematical Models and Methods in Bioengineering and Biosciences and the National Research Foundation of South Africa. The authors would like to thank the anonymous reviewers for their contributions to improving the quality of the paper.

\section{References}

[1] P. Hanusse, Existence of limited ring in development of open chemical systems, C. R. Acad. Sci. C Chim. 274 (1972) 1245-1247.

[2] J. Murray, Mathematical Biology II: Spatial Models and Biomedical Applications, third ed., Springer, New York, 2003.

[3] G. Nicolis, I. Prigogine, Self-organization in Nonequilibrium Systems, Wiley, New York, 1977.

[4] D.W. Peaceman, H.H. Rachford Jr., The numerical solution of parabolic and elliptic differential equations, J. SIAM 3 (1955) 28-41.

[5] I. Prigogine, R. Lefever, Symmetry breaking instabilities in dissipative systems II, J. Chem. Phys. 48 (1968) 1695-1700.

[6] S.M. Stoltz, Nonlinear Dynamics (webpage), http://linus.up.ac.za/academic/maths/ND/Index. html.

[7] J.J. Tyson, Some further studies of nonlinear oscillations in chemical systems, J. Chem. Phys. 58 (1973) 3919-3930.

[8] J.J. Tyson, J.C. Light, Properties of two-component bimolecular and trimolecular chemical reaction systems, J. Chem. Phys. 59 (1973) 4164-4173.

[9] P.H. William, Numerical Recipes: The Art of Scientific Computing, third edition, Cambridge University Press, 2007.

[10] L. Yang, M. Dolnik, A.M. Zhabotinsky, I.R. Epstein, Spatial resonances and superposition patterns in a reaction-diffusion model with interacting Turing modes, Phys. Rev. Lett. 88 (2002) 208303.

[11] L. Yang, A.M. Zhabotinsky, I.R. Epstein, Stable squares and other oscillatory Turing patterns in a reaction-diffusion model Phys. Rev. Lett. 92 (2004) 198303.

[12] Peter Rashkov, Regular and Discontinuous Solutions in a Reaction-Diffusion Model for Hair Follicle Spacing, Biomath 3 (2014), 1411111, http://dx.doi.org/10.11145/j.biomath.2014.11.111 